\title{
Sociodemographic and Psychological Predictors of Intention to Receive a COVID-19 Vaccine in Elderly Peruvians
}

\author{
Tomás Caycho-Rodríguez ${ }^{1}$ (1) . José M. Tomás ${ }^{2}$. Carlos Carbajal-León ${ }^{1}$. \\ Lindsey W. Vilca ${ }^{3}$. Mario Reyes-Bossio ${ }^{4}$. Claudio Intimayta-Escalante ${ }^{5}$. \\ Andrea Vivanco-Vidal ${ }^{4}$. Daniela Saroli-Araníbar ${ }^{4}$. \\ Renzo Felipe Carranza Esteban ${ }^{6} \cdot$ Michael White $^{7}$
}

Accepted: 3 August 2021 / Published online: 18 August 2021

(c) Associação Brasileira de Psicologia 2021

\begin{abstract}
The implementation of a vaccine against COVID-19 is one of the most important health strategies to mitigate the spread of the disease. The objective of this study was to estimate the prevalence of the intention to be vaccinated against COVID-19 and its predictors in older Peruvian adults. This is a cross-sectional study, where information was collected through an online survey regarding vaccination intention of the participants, as well as sociodemographic and psychological variables. A multiple regression analysis was applied to identify predictors of intention to be vaccinated against COVID-19. We evaluated 245 participants, who had a mean age of 72.74 years old $(\mathrm{SD}=6.66)$. $65.5 \%$ of these older adults expressed a high likelihood of accepting vaccination, while $20.9 \%$ expressed a low likelihood of accepting vaccination, and $13.6 \%$ were hesitant. Eleven predictors were identified that explained $66.69 \%$ of the intention to vaccinate against COVID-19. This identified place of residence, perceived likelihood of contracting COVID-19, severity of previous infection with COVID-19, fear of the disease, previous refusal of a vaccine, concerns about vaccine sales and speculation, and trust toward vaccines against COVID-19, as the main predictors. Our results show that confidence in vaccines and previous vaccine refusal are relevant predictors of intention to vaccinate against COVID-19 in older adults; these findings may be useful to guide the development of campaigns for the immunization of this vulnerable group in the current pandemic.
\end{abstract}

Keywords Elderly $\cdot$ COVID-19 $\cdot$ Intention to vaccinate $\cdot$ Peru

Tomás Caycho-Rodríguez tppcaycho@gmail.com

Extended author information available on the last page of the article 


\section{Introduction}

The pandemic caused by COVID-19 continues to have a major negative impact on public health and the economy globally (Thaker, 2021). There are currently (April 9, 2021) more than 133 million diagnosed cases and 2,894,664 million deaths in 192 countries (Coronavirus Resource Center, 2021). In Latin America (April 9, 2021), Peru is one of the countries most affected by COVID-19 with 1,607,898 million cases and 53,725 deaths (Ministerio de Salud, 2021). These figures are increasing rapidly. Although the disease affects people of any age, some groups, such as older adults, are at greater risk of presenting more severe symptoms and life-threatening complications, which is more likely in people with comorbidities, such as diabetes, hypertension, obesity and cardiovascular disease (AlvaradoSocarras et al., 2021). Currently in Peru, $13.0 \%$ of the Peruvian population is over 60 years of age (Instituto Nacional de Estadística e Informática [INEI], 2021) and this percentage is expected to increase to $15.7 \%$ by 2050 (INEI, 2016). According to estimates, to date (April 9, 2021) 37,397 older adults have died from COVID19, making them the age group most affected by the pandemic (Ministerio de Salud, 2021). Faced with this situation, countries have implemented strict actions to control the spread of COVID-19, which mainly include quarantine periods together with social distancing, mandatory use of masks and face shields (Qattan et al., 2021). However, such measures have not been sufficient, making the development and implementation of a vaccine one of the most important health strategies to mitigate the spread of this disease (Lurie et al., 2020).

Currently, more than 200 vaccines against COVID-19 are being developed worldwide (Dodd et al., 2021), where more than 93 are in clinical trials as of March 2021 (Tran et al., 2021) which are evaluating the efficacy and safety of each vaccine. Different studies have reported the effects of applying these vaccines in the population and the adequate immune response in many cases (Folegatti et al., 2020; Zhu et al., 2020). In this sense, several countries are securing agreements to access early doses of the vaccines. In the case of Peru, the government has finalized agreements with laboratories to guarantee the acquisition of, so far, 48 million doses: 1,000,000 Sinopharm vaccines, 20 million from Pfizer, 14 million from AstraZeneca and 13.2 million doses through the Covax Facility. Vaccine access is only one of the issues facing governments, as the success of vaccination strategies will depend on the vaccine reception among the population (Sherman et al., 2020). It is estimated that 70 to $80 \%$ of the population should be vaccinated to achieve herd immunity and limit community transmission of the virus (DeRoo et al., 2020; Kwok et al., 2020). However, vaccine refusal is considered to be one of the major global public health threats, even before the emergence of more cases and deaths due to COVID-19 (World Health Organization [WHO], 2019).

It was expected that the lethality of COVID-19 would generate high participation rates in vaccination programs (Ruiz \& Bell, 2021). Unfortunately, different studies indicate that some would not accept to receive the vaccine once it becomes available (Dodd et al., 2021; Neumann-Böhme et al., 2020). A global 
study involving 13,426 people from 19 countries indicated that approximately $71.5 \%$ of respondents would agree to receive a vaccine if it were proven safe and effective (Lazarus et al., 2021). However, other research indicates that vaccine acceptance rates vary globally (Wang et al., 2021). For example, in countries such as China, Brazil, South Africa, and South Korea, acceptance rates reached between 80 and 90\%; while in Russia and France it was only around 55\% and $60 \%$ respectively (Detoc et al., 2020; Lin et al., 2020a, b; Neumann-Böhme et al., 2020; Wang et al., 2020). In this context, it is vitally important to understand the factors associated with intention to receive a vaccine against COVID-19 in older adults, who are at higher risk of mortality and complications from COVID-19 infection (Kang \& Jung, 2020) and, thus, generally more likely to agree to be vaccinated against COVID-19 (Sherman et al., 2020). A study of adults over 65 years of age reported that 17.1 to $65.8 \%$ were very likely to accept vaccination against COVID-19 (Nguyen et al., 2021).

Previous studies have reported a set of sociodemographic and psychosocial factors associated with intention to be vaccinated against COVID-19 in the general population. Among the sociodemographic factors, those who mostly commonly show a higher intention to receive a COVID-19 vaccine have been males, older than 65 years of age, with higher household income and higher education. Likewise, having a spouse or partner and having previously received a vaccine were also associated with higher intention to be vaccinated; whereas place of residence was not significantly associated with intention to be vaccinated against COVID-19 (Al-Mohaithef \& Padhi, 2020; Meier et al., 2021). However, in other studies, it has also been reported that sociodemographic variables such as gender, age, educational level, number of people sharing a household, political ideology or religiosity were not significantly associated with intention to vaccinate (Berg \& Lin, 2020).

Based on the Health Belief Model (Rosenstock et al., 1988), research has been conducted to understand the psychosocial factors that explain the intention to be vaccinated against COVID-19 (Sherman et al., 2020; Wong et al., 2020, 2021). The Health Belief Model is a widely used theory for understanding health- and diseaserelated behaviors, comprising a set of constructs such as perceived susceptibility, perceived severity and benefits, perceived barriers, and self-efficacy to engage in a behavior (Glanz et al., 2008). Thus, studies indicate that perceived severity of COVID-19, perceived benefits of the vaccine, self-reported health status, trust in the health care system or vaccine manufacturers, greater knowledge about the vaccine, rejection of conspiracy theories about vaccines, having received vaccines previously, not relying on social networks for information about the virus and having a pre-existing illnesses were positively related to greater intention to be vaccinated against COVID-19 (Berg \& Lin, 2020; Ruiz \& Bell, 2021; Wong et al., 2020, 2021).

Therefore, due to the importance of vaccination in controlling the COVID-19 pandemic, it is important to identify factors associated with intention to vaccinate against COVID-19, especially in the populations that have been most affected by the pandemic, such as older adults. This is important because of declining confidence in vaccines (Allen et al., 2021) and in science in general (Donzelli et al., 2018), which has led to new COVID-19 vaccines being met with doubts about their efficacy and safety. Therefore, while governments make multisectoral efforts to distribute 
vaccines, the implementation of public health strategies that develop effective messages about vaccination campaigns is also necessary (Allen et al., 2021). Thus, the aim of this study was to identify predictors of intention to be vaccinated against COVID-19 in a sample of older Peruvian adults. Sociodemographic and psychological predictors were used, such as sex, educational level, fear of contracting the disease, speculation about vaccines, and confidence in the COVID-19 vaccine, among others. It is expected that the findings of the present study will allow for the development of effective strategies to improve the acceptance of the COVID-19 vaccine in older Peruvian adults.

\section{Method}

\section{Participants}

The study was cross-sectional. The a priori power analysis performed in the $\mathrm{G}^{*}$ Power program (Faul et al., 2009) using a small effect size (f2 $=0.15$ ), $\alpha=0.05$ and power $=0.80$, with twelve predictors indicated that 74 participants were sufficient to detect effects. However, the actual sample exceeded that number, as data was collected from 245 older adults. The inclusion criteria were as follows: people 60 years of age or older, residing in Peru and able to give informed consent to complete the survey. The demographic information of the participants is shown in Table 1.

\section{Instruments}

\section{Sociodemographic Variables}

A brief questionnaire was constructed to identify some sociodemographic characteristics of the participants, such as sex, age, income level, educational level, place of residence, marital status, children, employment status, whether the participant had COVID-19 and whether a family member or close friend had COVID-19, time exposed to information about COVID-19, frequency of leaving home, living with vulnerable people, refusal to be vaccinated in the past, and if they were suffering from a chronic disease.

\section{Attitudes Towards Vaccination}

The Vaccination Attitudes Examination (VAX) Scale (Martin \& Petrie, 2017) was used, which measures general negative attitudes toward vaccines. It is comprised of 12 items, which are scored on a six-point Likert-type scale ( $1=$ "Strongly Agree" to 6="Strongly Disagree"). The items are grouped into four dimensions: 1) distrust of vaccine benefit; 2) concern about unforeseen future effects; 3) concern about commercial effects and speculation; and 4) preference for natural immunity. The English version of the VAX was first translated into Spanish by two independent experts, and then translated back 
Table 1 Descriptive statistics for the variables and indicators in the study

\begin{tabular}{|c|c|c|}
\hline Quantitative variables or Indicators & Mean & SD \\
\hline Age & 72.74 & 6.66 \\
\hline Income level & 2.88 & 1.43 \\
\hline Hours thinking, seeing info about COVID & 1.59 & 0.90 \\
\hline How often you leave home & 2.68 & 1.20 \\
\hline Educational level & 1.22 & 1.39 \\
\hline How likely are you to get COVID? & 3.25 & 1.23 \\
\hline How likely are you to die if you get COVID? & 3.48 & 1.13 \\
\hline How severe is COVID? & 3.77 & 1.06 \\
\hline Are you worried about spreading COVID? & 3.47 & 0.75 \\
\hline What is the likelihood for you to vaccinate? & 3.78 & 1.47 \\
\hline Did you reject any vaccine as dangerous? & 1.92 & 0.27 \\
\hline Did you postpone any vaccine because it is dangerous? & 1.88 & 0.32 \\
\hline Have you received a vaccine even while hesitating? & 1.72 & 0.45 \\
\hline Anxiety & 1.57 & 1.76 \\
\hline Safe Behavior & 3.95 & 4.62 \\
\hline Fear of COVID & 14.80 & 5.74 \\
\hline Trust in the vaccine & 13.04 & 4.42 \\
\hline Worries of future unexpected side effects & 12.19 & 3.48 \\
\hline Worries on commerce and speculation & 9.35 & 4.56 \\
\hline Preference for natural immunity & 8.63 & 4.39 \\
\hline Categorical indicators & Categories & $\%$ \\
\hline \multirow[t]{2}{*}{ Sex } & Women & 43.6 \\
\hline & Men & 56.4 \\
\hline \multirow[t]{2}{*}{ Residence } & Lima & 68.16 \\
\hline & Other & 31.83 \\
\hline \multirow[t]{2}{*}{ Children } & Yes & 39.4 \\
\hline & No & 60.6 \\
\hline \multirow[t]{2}{*}{ Marital Status } & Married or couple & 30.4 \\
\hline & Others & 69.6 \\
\hline \multirow[t]{2}{*}{ Do you have a permanent job? } & Yes & 49.1 \\
\hline & No & 50.9 \\
\hline \multirow[t]{2}{*}{ Have you had COVID-19? } & Yes & 28.7 \\
\hline & No & 71.3 \\
\hline \multirow[t]{2}{*}{ Do you live with any vulnerable people? } & Yes & 68.5 \\
\hline & No & 31.5 \\
\hline \multirow[t]{2}{*}{ Have you refused a vaccine in the past? } & Yes & 8.7 \\
\hline & No & 91.3 \\
\hline \multirow[t]{2}{*}{ Area in which you live } & Rural & 5.9 \\
\hline & City & 94.1 \\
\hline \multirow[t]{2}{*}{ Cases of COVID in the family? } & Yes & 65.4 \\
\hline & No & 34.6 \\
\hline \multirow[t]{2}{*}{ Cases of COVID within your friends? } & Yes & 85.8 \\
\hline & No & 14.2 \\
\hline \multirow[t]{2}{*}{ Suffer from a chronic illness? } & Yes & 13.1 \\
\hline & No & 86.9 \\
\hline
\end{tabular}


into English. Subsequently, the two English versions were reviewed by two experts in the areas of public health. The content validity of the Spanish version of the VAX was evaluated by a group of 10 professionals in the fields of psychology, medicine, and epidemiology. Their analysis was based on the criteria of clarity, coherence and relevance with Aiken's V. It was concluded that the Spanish translation of the VAX was clear, coherent and relevant (all $V$ values were greater than 0.70 ). The reliability for each of the factors in the present study was: distrust in the benefit of the vaccine ( $\alpha=0.94)$, concern for unforeseen future effects $(\alpha=0.70)$, concern for commercial effects and speculation $(\alpha=0.87)$, and preference for natural immunity $(\alpha=0.88)$.

\section{Intention to be Vaccinated}

Intention to be vaccinated against COVID-19 was measured from the single item "How likely are you to decide to be vaccinated against COVID-19, if the vaccine were available?" which has been used in a previous study in the United States (Ruiz \& Bell, 2021). Response options are $1=$ not at all likely, $2=$ very unlikely, $3=$ unsure, $4=$ somewhat likely, and $5=$ very likely. Individuals who checked the "somewhat likely" or "very likely" responses were considered to be more susceptible to subsequent vaccination. The English version was translated into Spanish using the back-translation method. Content validity was assessed following the same procedure used with the VAX. Single-item measures to assess intention to vaccinate against COVID-19 have been used in other recent studies (Paul et al., 2021).

\section{Fear of COVID-19}

The Fear of COVID-19 Scale (FCV-19S; Ahorsu et al., 2020) was used, which aims to assess the fear of COVID-19 from seven items, which have a five-point Likerttype response scale $(1=$ strongly disagree to $5=$ strongly agree). The total score ranges from 7 to 35, where higher values indicate a greater fear of COVID-19. In this study, the version which has been validated for Peruvian older adults was used (Caycho-Rodriguez et al., 2021). The reliability of the FCV-19S in the current study was $\alpha=0.85$.

\section{Safety-Seeking Behaviors}

We used the Coronavirus Reassurance-Seeking Behaviors Scale (CRSBS; Lee et al, 2020), which is a self-report measure that assesses the frequency with which people perform reassurance-seeking behaviors. It is comprised of five items that have five response options $(0=$ not at all to $4=$ almost every day during the past 2 weeks $)$. A version translated into Spanish and validated in Peru was used in the present study (Caycho-Rodríguez et al., 2021). The reliability of the CRSBS was $\alpha=0.91$.

\section{Generalized Anxiety}

The Generalized Anxiety Disorder Scale (GAD-2; Kroenke et al., 2007) was used. The GAD-2 is a two-item self-report measure that assesses the severity of an 
individual's anxiety symptoms and was designed for use in primary care. Responses are given on a Likert-type scale (not at all $=0$, several days $=1$, more than half the days $=2$, and almost every day $=3$ ). The total score of the GAD-2 ranges from 0 to 6, where higher values are an indicator of a higher frequency of anxiety symptoms. In this study, the version validated in Peru (Merino-Soto et al., 2017) and used in research with older adults in the context of the COVID-19 pandemic (CaychoRodríguez et al., 2021) was used. In the present investigation, the GAD-2 presented adequate reliability $(\alpha=0.90)$.

\section{Other Measures}

The probability of contracting COVID-19 was assessed with the single item "I think my probability of contracting COVID-19 during the next 6 months is," with six response options $(1=$ practically nonexistent, $2=$ very small, $3=$ small, $4=$ large, $5=$ very large, $6=$ practically $100 \%$ ). Similarly, the severity of COVID19 was assessed with the item "How severe do you consider COVID-19 to be?" with five response options $(1=$ not at all severe, $2=$ somewhat severe, $3=$ severe, $4=$ quite severe, and $5=$ very severe). The question "How likely are you to die if you get COVID-19?" assesses the likelihood of dying from COVID-19 based on six response options $(1=$ virtually none, $2=$ very small, $3=$ small, $4=$ large, $5=$ very large, $6=$ virtually $100 \%$ ). Concern about transmitting COVID-19 was assessed with the question "How concerned are you about transmitting COVID-19 to another person?" which has four response options $(1=$ not at all, $2=$ somewhat, $3=$ quite a bit, and $4=$ very much). Refusing and postponing a vaccine because it was considered dangerous or ineffective were assessed with the questions "Have you ever refused a vaccine because you considered it dangerous?" and "Have you ever postponed a vaccine because of doubts about its efficacy?" respectively, which have dichotomous answers (Yes and No). Finally, receiving a vaccine despite doubts was assessed with the question "Have you ever received a vaccine even while doubting?" with two response options (Yes and No). All these questions were constructed specifically for this study.

\section{Procedure}

Participants were selected by non-probability snowball sampling. When a person was identified as meeting the inclusion criteria, he or she was asked to suggest other older adults interested in participating in the research study. In this sense, the telephone numbers of the potential participants served as a means of contact. Once it was verified that the person met the necessary criteria, their e-mail address or that of a close relative was requested in order to send the online survey. An online questionnaire was constructed, using the Google Forms platform, which served to collect the information for the study between January 3 and 31, 2021. In this sense, the collection of information began three days after the announcement of the purchase of the first batch of vaccines (January 6, 2021) and ended before the arrival in Peru of the first batch of Sinopharm vaccines (February 7, 2021). 
Online informed consent was obtained from participants prior to answering the questions. This informed consent provided participants with two options: "Yes" for those who wished to participate in the research study and "No" for those who did not wish to participate. Only older adults who selected "Yes" were able to access the following questions to complete the questionnaire. Likewise, all participants were informed of the objective of the study, the possibility of withdrawing from the study at any time, in addition to the anonymous and confidential nature of the information provided. The study was approved by the Ethics Committee of the Universidad Privada del Norte (Registration number: 20213002).

\section{Statistical Analyses}

The statistical analyses were performed in the software R Studio v4.0 (R Core Team, 2020). Descriptive statistics including means, standard deviations for (semi) quantitative variables and relative frequencies for categorical variables were calculated. Additionally, in order to analyze the bivariate relations of intention to be vaccinated, the response variable, with all the potential predictors, correlations were estimated with the semi-quantitative and quantitative variables and indicators, and independent samples $t$-tests with the binary indicators. Then multiple regression analyses were performed. Given the 31 variables evaluated as predictors, we employed the function "regsubsets" with the method "seqrep" from the "leaps" R-package (Lumley, 2020). This function and method chooses, from all the potential multiple regressions with 31 possible predictors, the best performing according to either the adjusted R-square, Bic or Cp values. We chose the number of predictors according to the adjusted $R$-square criteria. Residuals were analyzed to test for departure from model assumptions. Standardized estimates in the regression model were estimated using the QuantPsyc package, in the R library (Fletcher, 2012). All analyses were performed considering a 95\% confidence interval $(95 \% \mathrm{CI})$ and a significant $\mathrm{p}$-value of less than $0.05(p<0.05)$.

\section{Results}

\section{Descriptive and Bivariate Analyses}

Descriptive analysis regarding intention to be vaccinated against COVID-19 identified that $12.5 \%$ of participants did not consider it likely that they would be vaccinated against COVID-19, $8.4 \%$ considered it very unlikely to be vaccinated, $13.6 \%$ reported feeling unsure about being vaccinated, while $65.5 \%$ indicated high likelihood of being vaccinated if COVID-19 vaccine were available.

Correlations between the intention to be vaccinated and the various potential predictors have been calculated, and a number of them were statistically significant $(p<0.05)$ and positively associated: 0.14 with hours thinking or receiving information about COVID-19; 0.19 with the perceived likelihood to get COVID-19; 0.12 with perceived likelihood of dying from COVID-19; 0.41 with the perceived severity of COVID-19; 0.33 with the worries to spread COVID-19; 0.21 with having 
previously postponed a vaccination; 0.21 with anxiety; 0.24 with fear of COVID-19; and 0.76 with the trust in the vaccines.

There were three negative and statistically significant relations with intention to be vaccinated: -0.31 with worries of future unexpected side effects; -0.57 with worries on commerce and speculation; and -0.42 with preference for natural immunity. The rest of correlations with age, educational level, income, frequency of going out and having received a vaccine even while hesitating were not statistically significant $(p \geq 0.05)$.

Independent samples $t$-tests were performed for comparing means of intention to be vaccinated in the binary indicators. Most comparisons were not statistically significant $(p \geq 0.05)$ : sex, marital status, permanent work, having had COVID-19, being in charge of a vulnerable person, living in a city or a rural area, having had a relative or friend affected with COVID-19, and having chronic illnesses. However, three t-tests were statistically significant. The participants living in Lima had more intention to vaccinate $(M=3.93)$ than participants from other parts of the country $(M=3.43, t(287)=-2.71, p=0.007)$. Finally, there are clear and large mean differences between those who have rejected a vaccine $(M=1.60)$ and those who have not $(M=3.98, t(287)=8.66, p<0.001)$.

\section{Regression Analyses}

We applied a stepwise procedure to select the best-fitting model in terms of proportion of variance explained, as already mentioned in the method section. This procedure selected a total of 11 predictors among the 31 available. This regression model was statistically significant $(F[11,277]=53.41, p<0.001)$, and adjusted $R$-square was 0.667 . That indicates that $66.7 \%$ of the variance in intention to vaccinate was explained by the 11 predictors. Estimates for the intercept and the 11 predictors are presented in Table 2 .

As can be seen in Table 2, place of residence had a positive and marginally significant effect on the intention to be vaccinated, with residents in Lima more willing to be vaccinated. On the other hand, perceived probability to get COVID-19, its severity and the fear it produces were all positively and statistically significantly related with the intention to be vaccinated ( $\beta$ values of $0.10,0.14$ and 0.07 ; respectively). Two predictors were negatively and significantly related to the response variable. Those who refused a vaccine in the past had less intention to be vaccinated $(\beta=-0.45)$, and the same happened with those with more worries on commerce and speculation with the vaccines $\beta=-0.22$ ). However, the main predictor of the intention to vaccinate happened to be the trust in the vaccines for COVID-19 $\beta=0.55$ ).

\section{Discussion}

Vaccines are considered an important public health strategy to stop the spread of COVID-19 (Harapan et al., 2020). However, in the current context, assessing the factors that predict the intention to be vaccinated against COVID-19 is 
Table 2 Regression model estimates and their significance

\begin{tabular}{lcccccc}
\hline Predictor & $B$ & $\beta$ & SE & $95 \%$ CI & $t$ & $p$ \\
\hline Intercept & 0.75 & & 0.35 & $0.06,1.44$ & 2.14 & .033 \\
Residence & 0.21 & & 0.10 & $-0.01,0.42$ & 1.93 & .054 \\
Having children & -0.17 & & 0.10 & $-0.38,0.02$ & -1.70 & .088 \\
Refused vaccination in the past & -0.45 & & 0.21 & $-0.86,-0.03$ & -2.14 & .032 \\
Chronic diseases & -0.21 & & 0.15 & $-0.51,0.07$ & -1.43 & .153 \\
Perceived probability to get COVID & 0.11 & .10 & 0.04 & $0.03,0.20$ & 2.82 & .005 \\
Perceived severity of COVID & 0.18 & .14 & 0.05 & $0.07,0.28$ & 3.41 & $<.001$ \\
Fear of COVID & 0.01 & .07 & 0.00 & $0.01,0.03$ & 1.97 & 0.048 \\
Trust in the vaccine & 0.17 & .55 & 0.01 & $0.14,0.20$ & 11.71 & $<.001$ \\
Worries on commerce and speculation & -0.06 & -.22 & 0.01 & $-0.09,-0.02$ & -3.87 & $<.001$ \\
Income & 0.04 & .03 & 0.03 & $-0.02,0.11$ & 1.25 & 0.210 \\
Preference for natural immunity & -0.01 & .06 & 0.01 & $-0.04,0.01$ & -1.14 & 0.254 \\
\hline
\end{tabular}

Note: Standardized estimates only for quantitative variables

very important for immunization programs. This is especially true in older adults because they are a group at high risk of mortality from COVID-19 (Kang \& Jung, 2020). In that sense, the results indicate that, if the COVID-19 vaccine were available, the majority of older adults $(65.5 \%)$ expressed a high likelihood of accepting a vaccine, indicating that the majority of participants support vaccination against COVID-19. This result is not surprising, as data collection began when the number of diagnosed cases and deaths from COVID-19 was on a significant rise in Peru. In January alone, 1884 deaths were counted and with more than 4000 cases diagnosed daily on average, reaching a peak of 15,792 confirmed cases on January 15 (Ministries of Health, 2021). This percentage is lower than the $71.5 \%$ reported in the general population of 19 countries (Lazarus et al., 2021). However, it is higher than the $39.4 \%$ reported in US adults over 65 years of age (Nguyen et al., 2021). The difference between the percentages of participants who intended to be vaccinated may be explained in part by the different timing of the studies, the difference in data collection, sociodemographic characteristics, and the way in which the question about intention to vaccinate was asked. There was also a significant percentage of older adults $(20.9 \%)$ who expressed low probability of receiving the vaccine, and $13.6 \%$ were hesitant due to their uncertainty with the vaccine. The latter could suggest a possible unequal acceptance of the vaccine in this at-risk population group. It is important to consider that a person's intention to be vaccinated is not necessarily a good predictor of the final decision to be vaccinated, which is determined by different factors and may vary over time, especially with the increase of vaccinated people in the environment (Lazarus et al., 2021). However, it is possible that the increase or decrease of diagnosed cases and deaths from COVID-19 may affect the intention to vaccinate (Motta, 2021). Therefore, constant monitoring of the opinion that older adults have about the vaccine and their participation in vaccination programs may allow for identifying variations in vaccination intentions that could modify the 
results obtained from this research study and improve immunization programs for each specific context.

In the predictive model, place of residence positively and statistically significantly predicted intention to be vaccinated against COVID-19. In this sense, participants living in Lima had a higher intention to be vaccinated than participants from other parts of the country. This finding is understandable, since inhabitants of regions or cities with higher rates of conformed cases of COVID-19 have greater fear and awareness of this disease (Fitzpatrick et al., 2020), which is associated with a higher probability of receiving a vaccine (Schmid et al., 2017). In that sense, the city of Lima concentrates the highest number of positive diagnoses and deaths due to COVID-19 since the beginning of the pandemic (Web Open COVID).

On the other hand, older adults who are more fearful of COVID-19, who perceive a greater likelihood of contracting COVID-19 or greater severity of the disease express a greater intention to be vaccinated against COVID-19. These findings are similar to what has been reported previously in populations other than older adults (Lin et al., 2020a, b; Reiter et al., 2018). Beliefs about the likelihood of contracting COVID-19 and perceived severity are important constructs within different theoretical models of health behavior, such as the health belief model (Rosenstock et al., 1988), and have shown relationships with acceptance of other vaccines, such as influenza (Ling et al., 2019). Previous studies indicate that assessing the perception of increased susceptibility or high risk regarding the disease would help further preventive actions to be developed and epidemic control to be improved (Verelst et al., 2016). Therefore, future interventions may aim to modify these beliefs, as suggested by previous interventions that have shown success in improving the acceptance of other vaccines (Reiter et al., 2018).

Those older adults who had refused vaccination in the past had less intention to be vaccinated. This result may be due to a set of factors such as doubts about the real need for vaccination and overexposure of the immune system, as well as perception about the safety and efficacy of vaccines or previous negative experiences with vaccines (Succi, 2018). Likewise, this result is of concern as prior refusal to receive a vaccine may increase the risk of contracting preventable diseases with high mortality (Bianco et al., 2019). Similarly, people's attitudes towards vaccines are an expected predictor of intention to vaccinate (Myers \& Goodwin, 2011). Thus, those who had more concerns about trade and speculation with vaccines for COVID-19 also had less intention to be vaccinated. This finding provides further support for previous literature demonstrating that anti-vaccine attitudes are inversely related to intention to vaccinate for COVID-19, as reported by studies in general populations in the UK (Paul et al., 2021) and Turkey (Huynh \& Senger, 2021). Concern about commercial speculation of vaccines expresses the fear of people, in this case older adults, about the influence that pharmaceutical companies have on the development and dissemination of vaccines (Martin \& Petrie, 2017). In this sense, people who maintain this attitude towards vaccination tend to worry about the presence of ineffective vaccines related to the economic benefits that pharmaceutical companies have obtained in their development, which predicts a lower intention to be vaccinated (Huynh, \& Senger, 2021). 
The predictive model also indicated that the main predictor of intention to be vaccinated in older adults was vaccine confidence. Thus, the older adults who were less likely to be vaccinated reported lower confidence in the COVID-19 vaccine. This result is similar to that reported in previous studies, which suggest that low confidence in vaccines is an important barrier to greater intention to be vaccinated against COVID-19 (Paul et al., 2021; Sherman et al., 2020). In recent months, Peru has experienced different negative situations associated with vaccination scandals and malpractices that have spread a loss of confidence in vaccines by the general population (Kenyon, 2021), which probably affects the results of this research study. Therefore, health institutions that watch over public health and the scientific community should develop campaigns aimed at promoting vaccination against COVID19 , focused on increasing confidence in vaccines, in families and in those at-risk groups such as older adults who are undecided or unwilling to receive the vaccine. For this, adequate information about this new disease, as well as the importance of vaccines and possible side effects, should be provided. In this sense, it is important to emphasize the transparency of information and respond to the different concerns people have about the efficacy and safety of vaccines with regards to their rapid development (Saied et al., 2021).

\section{Limitations}

Our study has limitations that are important to note. First, the cross-sectional design only allowed us to assess intention to be vaccinated at a single point in time; therefore, we cannot account for variations in intention to be vaccinated in response to other changes, such as the increase in diagnosed cases and deaths from COVID19 , the increased dissemination of evidence on vaccine safety, or access to vaccines (Wang et al., 2021). In this sense, it is important to remember that the information was collected before the arrival of the first batch of vaccines to Peru, and older adults, along with the general population, did not have enough information about the safety and effectiveness of these vaccines. Thus, intentions to be vaccinated may change after the vaccination process has begun. Second, due to the use of a nonprobability snowball sample, the study sample was not fully representative of Peruvian older adults. Therefore, the results cannot be generalized to other older adults in different regions of Peru. Third, the use of a Google Form to collect information led to the participation of only older adults with Internet access, which would limit the representativeness of the sample. Fourth, intention to be vaccinated was investigated, but actual vaccination behavior is likely to be modified by the change in context (Sherman et al., 2020). However, because of the importance of intention within theories of health behavior adoption, factors associated with intention to be vaccinated in this research study would also be expected to influence vaccination behavior. Similarly, intention to be vaccinated was assessed based on a hypothetical vaccine, which may be different from the intentions reported by participants in a real situation. Fifth, despite the diversity of sociodemographic characteristics, it is likely that some subgroups of older adults were not fully represented. In addition, the binary representation of some variables may have led to an oversimplification 
of these categories. Sixth, the data collected were self-reported, which may have resulted in recall bias and participants reporting a greater intention to be vaccinated due to social desirability. Additionally, some older adults required assistance to be able to perform their daily activities so, in that sense, discrepancies about the importance of COVID-19 vaccination in family caregivers and older adults may determine participation in immunization programs.

\section{Conclusions}

In conclusion, Peruvian older adults reported a high intention to be vaccinated against COVID-19. Also, intention to be vaccinated can be predicted by place of residence, perceived likelihood of contracting COVID-19, previous refusal of a vaccine, concerns about trade or speculation with vaccines, and trust towards vaccines, the latter being the main predictor. Thus, future efforts to improve the vaccination process of older adults should address some of the variables mentioned above by implementing evidence-based interventions (Williams et al., 2020). Still, additional studies should include measures of fear of vaccine side effects and other variables important for understanding intention to be vaccinated against COVID-19. (Petravić et al., 2021).

The results may be useful to guide the development of innovative campaigns to promote the intention to be vaccinated against COVID-19, or other future epidemics, in the older adult group, prior to the implementation of mass vaccination campaigns. In this regard, as mentioned above, campaigns should provide transparent and truthful information about vaccines, since older adults' confidence in the safety and efficacy of vaccines is related to their intention to receive a vaccine against COVID-19. To build confidence in COVID-19 vaccines, messages should focus on the benefits of vaccines for the entire population, including older adults. Thus, for example, displaying positive information about COVID-19 vaccination enhances positive perceptions associated with vaccination, which in turn increases behavioral intention to be vaccinated against the disease (Zhang et al., 2021). However, longitudinal studies are needed to test this relationship. In addition to sharing information on vaccine safety, continuous monitoring of side effects of COVID-19 vaccines should be widely reported to strengthen the confidence of older adults (Ogilvie et al., 2021). Also, in addition to broader information campaigns, efforts should be made to facilitate communication between older adults and health care providers about the safety and efficacy of COVID19 vaccines (Nikolovski et al., 2021). Interventions could be done by combining different media (television, radio, print, social media), where broadcasters and journalists help to administer consistent messages and go against misinformation (Davis et al., 2020). It is also suggested to explicitly draw on existing intervention messages, such as "We're in this together" (Williams et al., 2020). These efforts are expected to generate more rapid acceptance of vaccination. The importance of increasing acceptance rates of COVID-19 vaccination in older adults should not be underestimated, as increasing the number of vaccinated individuals, along with the presence of other preventive behaviors, will decrease community 
transmission rates, allow for herd immunity and a gradual return to normal activities (Kecojevic et al., 2021).

Author Contribution All authors contributed to the study conception and design. Material preparation and data collection were performed by TC-R, CC-L, DS-A, MR-B, AV-V, CI-E, RFCE, and MW. Data analysis was performed by JMT and LWV. The first draft of the manuscript was written by TC-R and all authors commented on previous versions of the manuscript. All authors read and approved the final manuscript.

Funding This research was funded by Universidad Privada del Norte.

Data Availability The database is available with a request to the corresponding author.

Code Availability Does not apply.

\section{Declarations}

Ethics Approval This study was performed in line with the principles of the Declaration of Helsinki. Approval was granted by the Ethics Committee of Universidad Privada del Norte (Registration number: 20213002).

Consent to Participate Informed consent was obtained from all individual participants included in the study.

Consent for Publication Does not apply.

Conflict of Interest The authors declare no competing interests.

\section{References}

Ahorsu, D. K., Lin, C. Y., Imani, V., Saffari, M., Griffiths, M. D., \& Pakpour, A. H. (2020). The fear of COVID-19 scale: Development and initial validation. International Journal of Mental Health and Addiction, 1-9,. https://doi.org/10.1007/s11469-020-00270-8

Allen, J. D., Abuelezam, N. N., Rose, R., \& Fontenot, H. B. (2021). Factors associated with the intention to obtain a COVID-19 vaccine among a racially/ethnically diverse sample of women in the USA. Translational Behavioral Medicine, 11(3), 785-792. https://doi.org/10.1093/tbm/ibab014

Al-Mohaithef, M., \& Padhi, B. K. (2020). Determinants of COVID-19 vaccine acceptance in Saudi Arabia: A web-based national survey. Journal of Multidisciplinary Healthcare, 13, 1657-1663. https:// doi.org/10.2147/JMDH.S276771

Alvarado-Socarras, J. L., Vesga-Varela, A. L., Quintero-Lesmes, D. C., Fama-Pereira, M. M., SerranoDiaz, N. C., Vasco, M., ..., Rodriguez-Morales, A. J. (2021). Perception of COVID-19 vaccination amongst physicians in Colombia. Vaccines, 9(3), 287. https://doi.org/10.3390/vaccines9030287

Becker, M. H. (1974). The health belief model and sick role behavior. Health Education Monographs, 2(4), 409-419. https://doi.org/10.1177/109019817400200407

Berg, M. B., \& Lin, L. (2020). Prevalence and predictors of early COVID-19 behavioral intentions in the United States. Translational Behavioral Medicine, 10(4), 843-849. https://doi.org/10.1093/tbm/ ibaa085

Bianco, A., Mascaro, V., Zucco, R., \& Pavia, M. (2019). Parent perspectives on childhood vaccination: How to deal with vaccine hesitancy and refusal? Vaccine, 37(7), 984-990. https://doi.org/10.1016/j. vaccine.2018.12.062

Caycho-Rodríguez, T., Tomás, J. M., Barboza-Palomino, M., Ventura-León, J., Gallegos, M., ReyesBossio, M., \& Vilca, L. W. (2021). Assessment of Fear of COVID-19 in Older Adults: Validation of 
the Fear of COVID-19 Scale. International Journal of Mental Health and Addiction, 1-15,. https:// doi.org/10.1007/s11469-020-00438-2

Coronavirus Resource Center. (2021). COVID-19 Dashboard by the Center for Systems Science and Engineering (CSSE) at Johns Hopkins University (JHU). https://coronavirus.jhu.edu/map.html. Accessed 9 Apr 2021.

DeRoo, S. S., Pudalov, N. J., \& Fu, L. Y. (2020). Planning for a COVID-19 vaccination program. JAMA, 323(24), 2458-2459. https://doi.org/10.1001/jama.2020.8711

Detoc, M., Bruel, S., Frappe, P., Tardy, B., Botelho-Nevers, E., \& Gagneux-Brunon, A. (2020). Intention to participate in a COVID-19 vaccine clinical trial and to get vaccinated against COVID-19 in France during the pandemic. Vaccine, 38(45), 7002-7006. https://doi.org/10.1016/j.vaccine.2020. 09.041

Dodd, R. H., Pickles, K., Nickel, B., Cvejic, E., Ayre, J., Batcup, C., .., McCaffery, K. J. (2021). Concerns and motivations about COVID-19 vaccination. The Lancet. Infectious Diseases, 21(2), 161163. https://doi.org/10.1016/S1473-3099(20)30926-9

Donzelli, G., Palomba, G., Federigi, I., Aquino, F., Cioni, L., Verani, M., ..., Lopalco, P. (2018). Misinformation on vaccination: A quantitative analysis of YouTube videos. Human Vaccines \& Immunotherapeutics, 14(7), 1654-1659. https://doi.org/10.1080/21645515.2018.1454572

Faul, F., Erdfelder, E., Buchner, A., \& Lang, A. G. (2009). Statistical power analyses using G* Power 3.1: Tests for correlation and regression analyses. Behavior Research Methods, 41(4), 1149-1160. https://doi.org/10.3758/BRM.41.4.1149

Fitzpatrick, K. M., Harris, C., \& Drawve, G. (2020). Fear of COVID-19 and the mental health consequences in America. Psychological Trauma: Theory, Research, Practice, and Policy, 12(S1), S17S21. https://doi.org/10.1037/tra0000924

Fletcher, T. D. (2012). QuantPsyc: Quantitative Psychology Tools. R package version 1.5. https:// CRAN.R-project.org/package=QuantPsyc. Accessed 10 Mar 2021.

Folegatti, P. M., Ewer, K. J., Aley, P. K., Angus, B., Becker, S., Belij-Rammerstorfer, S., ..., Hamlyn, J. (2020). Safety and immunogenicity of the ChAdOx1 nCoV-19 vaccine against SARS-CoV-2: A preliminary report of a phase 1/2, single-blind, randomised controlled trial. The Lancet, 396(10249), 467-478. https://doi.org/10.1016/S0140-6736(20)31604-4

García, L. Y., \& Cerda, A. A. (2020). Contingent assessment of the COVID-19 vaccine. Vaccine, 38(34), 5424-5429. https://doi.org/10.1016/j.vaccine.2020.06.068

Glanz, K., Rimer, B. K., \& Viswanath, K. (Eds.). (2008). Health behavior and health education: theory, research, and practice. Wiley.

Harapan, H., Wagner, A. L., Yufika, A., Winardi, W., Anwar, S., Gan, A. K., .., Mudatsir, M. (2020). Acceptance of a COVID-19 vaccine in southeast Asia: A cross-sectional study in Indonesia. Frontiers in Public Health, 8, 381. https://doi.org/10.3389/fpubh.2020.00381

Huynh, H. P., \& Senger, A. R. (2021). A little shot of humility: Intellectual humility predicts vaccination attitudes and intention to vaccinate against COVID-19. Journal of Applied Social Psychology, 51(4), 449-460. https://doi.org/10.1111/jasp.12747

Instituto Nacional de Estadística e Informática. (2016). Perú: estimaciones y proyecciones de población 1950-2050. Lima: Instituto Nacional de Estadística e Informática. https://www.inei.gob.pe/media/ MenuRecursivo/publicaciones_digitales/Est/Lib0466/Libro.pdf. Accessed 9 Apr 2021.

Instituto Nacional de Estadística e Informática. (2021). Situación de la población adulta mayor. Informe Técnico. Lima: Instituto Nacional de Estadística e Informática. https://www.inei.gob.pe/media/ MenuRecursivo/boletines/02-informe-tecnico-poblacion-adulta-mayor-ene-feb-mar-2021.pdf. Accessed 9 Apr 2021.

Kang, S. J., \& Jung, S. I. (2020). Age-related morbidity and mortality among patients with COVID-19. Infection \& Chemotherapy, 52(2), 154-164. https://doi.org/10.3947/ic.2020.52.2.154

Kecojevic, A., Basch, C. H., Sullivan, M., Chen, Y. T., \& Davi, N. K. (2021). COVID-19 Vaccination and Intention to Vaccinate Among a Sample of College Students in New Jersey. Journal of Community Health, 1-10,. https://doi.org/10.1007/s10900-021-00992-3

Kenyon, G. (2021). Vacuna-gate escalates in Peru. The Lancet Infectious Diseases, 21(4), 463. https://doi. org/10.1016/S1473-3099(21)00157-2

Kroenke, K., Spitzer, R. L., Williams, J. B., Monahan, P. O., \& Löwe, B. (2007). Anxiety disorders in primary care: Prevalence, impairment, comorbidity, and detection. Annals of Internal Medicine, 146(5), 317-325. https://doi.org/10.7326/0003-4819-146-5-200703060-00004 
Kwok, K. O., Lai, F., Wei, W. I., Wong, S. Y. S., \& Tang, J. W. (2020). Herd immunity-estimating the level required to halt the COVID-19 epidemics in affected countries. Journal of Infection, 80(6), e32-e33. https://doi.org/10.1016/j.jinf.2020.03.027

Lazarus, J. V., Ratzan, S. C., Palayew, A., Gostin, L. O., Larson, H. J., Rabin, K., ..., El-Mohandes, A. (2021). A global survey of potential acceptance of a COVID-19 vaccine. Nature Medicine, 27(2), 225-228. https://doi.org/10.1038/s41591-020-1124-9

Lee, S., Jobe, M., Mathis, A., \& Gibbons, J. (2020). Incremental validity of coronaphobia: Coronavirus anxiety explains depression, generalized anxiety, and death anxiety. Journal of Anxiety Disorders, 74, 1-4. https://doi.org/10.1016/j.janxdis.2020.102268

Lin, Y., Hu, Z., Zhao, Q., Alias, H., Danaee, M., \& Wong, L. P. (2020a). Understanding COVID-19 vaccine demand and hesitancy: A nationwide online survey in China. PLoS Neglected Tropical Diseases, 14(12), e0008961. https://doi.org/10.1371/journal.pntd.0008961

Lin, Y., Hu, Z., Zhao, Q., Alias, H., Danaee, M., \& Wong, L. P. (2020b). Understanding COVID-19 vaccine demand and hesitancy: A nationwide online survey in China. PLoS Neglected Tropical Ddiseases, 14(12), e0008961. https://doi.org/10.1371/journal.pntd.0008961

Ling, M., Kothe, E. J., \& Mullan, B. A. (2019). Predicting intention to receive a seasonal influenza vaccination using Protection Motivation Theory. Social Science \& Medicine, 233, 87-92. https://doi.org/ 10.1016/j.socscimed.2019.06.002

Lumley, T. (2020). Leaps: Regression subset selection. $R$ package version 3.1. https://CRAN.Rproject. org/package=leaps. Accessed 10 Mar 2021.

Lurie, N., Saville, M., Hatchett, R., \& Halton, J. (2020). Developing Covid-19 vaccines at pandemic speed. New England Journal of Medicine, 382(21), 1969-1973. https://doi.org/10.1056/NEJMp 2005630

Martin, L. R., \& Petrie, K. J. (2017). Understanding the dimensions of anti-vaccination attitudes: The vaccination attitudes examination (VAX) scale. Annals of Behavioral Medicine, 51(5), 652-660. https://doi.org/10.1007/s12160-017-9888-y

Meier, B. P., Dillard, A. J., \& Lappas, C. M. (2021). Predictors of the intention to receive a SARS-CoV-2 vaccine. Journal of Public Health. fdab013. https://doi.org/10.1093/pubmed/fdab013.

Ministerio de Salud. (2021). Sala situacional Covid-19 Perú. https://covid19.minsa.gob.pe/sala_situa cional.asp. Accessed 9 Apr 2021.

Motta, M. (2021). Can a COVID-19 vaccine live up to Americans' expectations? A conjoint analysis of how vaccine characteristics influence vaccination intentions. Social Science \& Medicine, 272, 113642. https://doi.org/10.1016/j.socscimed.2020.113642

Myers, L. B., \& Goodwin, R. (2011). Determinants of adults' intention to vaccinate against pandemic swine flu. BMC Public Health, 11(1), 1-8. https://doi.org/10.1186/1471-2458-11-15

Neumann-Böhme, S., Varghese, N. E., Sabat, I., Barros, P. P., Brouwer, W., van Exel, J., ..., Stargardt, T. (2020). Once we have it, will we use it? A European survey on willingness to be vaccinated against COVID-19. The European Journal of Health Economics, 21, 977-982. https://doi.org/10. 1007/s10198-020-01208-6

Nikolovski, J., Koldijk, M., Weverling, G. J., Spertus, J., Turakhia, M., Saxon, L., ..., Navar, A. M. (2021). Factors indicating intention to vaccinate with a COVID-19 vaccine among older US Adults. PLoS ONE, 16(5), e0251963. https://doi.org/10.1371/journal.pone.0251963

Nguyen, K. H., Srivastav, A., Razzaghi, H., Williams, W., Lindley, M. C., Jorgensen, C., ..., Singleton, J. A. (2021). COVID-19 vaccination intent, perceptions, and reasons for not vaccinating among groups prioritized for early vaccination-United States, September and December 2020. American Journal of Transplantation, 21(4), 1650-1656. https://doi.org/10.1111/ajt.16560

Ogilvie, G. S., Gordon, S., Smith, L. W., Albert, A., Racey, C. S., Booth, A., ..., Sadarangani, M. (2021). Intention to receive a COVID-19 vaccine: Results from a population-based survey in Canada. $B M C$ Public Health, 21(1), 1-14. https://doi.org/10.1186/s12889-021-11098-9

Petravić, L., Arh, R., Gabrovec, T., Jazbec, L., Rupčić, N., Starešinič, N., ..., Slavec, A. (2021). Factors affecting attitudes towards COVID-19 vaccination: An online survey in Slovenia. Vaccines, 9(3), 247. https://doi.org/10.3390/vaccines9030247.

Qattan, A., Alshareef, N., Alsharqi, O., Al Rahahleh, N., Chirwa, G. C., \& Al-Hanawi, M. K. (2021). Acceptability of a COVID-19 vaccine among healthcare workers in the Kingdom of Saudi Arabia. Frontiers in Medicine, 8, 83. https://doi.org/10.3389/fmed.2021.644300

R Core Team. (2020). R: A language and environment for statistical computing. R Foundation for Statistical Computing, Vienna, Austria. https://www.R-project.org/. Accessed 10 Mar 2021. 
Reiter, P. L., Katz, M. L., Bauermeister, J. A., Shoben, A. B., Paskett, E. D., \& McRee, A. L. (2018). Increasing human papillomavirus vaccination among young gay and bisexual men: A randomized pilot trial of the outsmart HPV intervention. LGBT Health, 5(5), 325-329. https://doi. org/10.1089/lgbt.2018.0059

Rogers, R. W. (1983). Cognitive and physiological processes in fear appeals and attitude change: A revised theory of protection motivation. In J. T. Cacioppo \& R. E. Petty (Eds.), Social Psychophysiology (pp. 153-176). Guilford Press.

Rosenstock, I. M., Strecher, V. J., \& Becker, M. H. (1988). Social learning theory and the health belief model. Health Education Quarterly, 15(2), 175-183. https://doi.org/10.1177/109019818801500203

Ruiz, J. B., \& Bell, R. A. (2021). Predictors of intention to vaccinate against COVID-19: Results of a nationwide survey. Vaccine, 39(7), 1080-1086. https://doi.org/10.1016/j.vaccine.2021.01.010

Saied, S. M., Saied, E. M., Kabbash, I. A., \& Abdo, S. A. E. F. (2021). Vaccine hesitancy: Beliefs and barriers associated with COVID-19 vaccination among Egyptian medical students. Journal of Medical Virology. https://doi.org/10.1002/jmv.26910

Schmid, P., Rauber, D., Betsch, C., Lidolt, G., \& Denker, M. L. (2017). Barriers of influenza vaccination intention and behavior-a systematic review of influenza vaccine hesitancy, 2005-2016. PLOS ONE, 12(1), e0170550. https://doi.org/10.1371/journal.pone.0170550

Sherman, S. M., Smith, L. E., Sim, J., Amlôt, R., Cutts, M., Dasch, H., ..., Sevdalis, N. (2020). COVID-19 vaccination intention in the UK: Results from the COVID-19 vaccination acceptability study (CoVAccS), a nationally representative cross-sectional survey. Human Vaccines \& Immunotherapeutics, 1-10, https://doi.org/10.1080/21645515.2020.1846397

Succi, R. C. D. M. (2018). Vaccine refusal-what we need to know. Jornal De Pediatria, 94(6), 574581. https://doi.org/10.1016/j.jped.2018.01.008

Thaker, J. (2021). The Persistence of Vaccine Hesitancy: COVID-19 Vaccination Intention in New Zealand. Journal of Health Communication, 1-8,. https://doi.org/10.1080/10810730.2021.1899346

Tran, V. D., Pak, T. V., Gribkova, E. I., Galkina, G. A., Loskutova, E. E., Dorofeeva, V. V., ..., Pham, D. T. (2021). Determinants of COVID-19 vaccine acceptance in a high infection-rate country: a crosssectional study in Russia. Pharmacy Practice, 19(1), 2276. https://doi.org/10.18549/PharmPract. 2021.1.2276.

Verelst, F., Willem, L., \& Beutels, P. (2016). Behavioural change models for infectious disease transmission: A systematic review (2010-2015). Journal of the Royal Society Interface, 13(125), 20160820. https://doi.org/10.1098/rsif.2016.0820

Vivanco-Vidal, A., Saroli-Araníbar, D., Caycho-Rodríguez, T., Carbajal-León, C., Barboza-Palomino, M., \& Reyes-Bossio, M. (2021). Evidencia de validez y confiabilidad de la versión en español de la Coronavirus Reassurance-Seeking Behaviors Scale en adultos de Lima, Perú. Ansiedad y Estrés. In press.

Wang, J., Jing, R., Lai, X., Zhang, H., Lyu, Y., Knoll, M. D., \& Fang, H. (2020). Acceptance of COVID19 Vaccination during the COVID-19 Pandemic in China. Vaccines, 8(3), 482. https://doi.org/10. 3390/vaccines 8030482

Wang, J., Lu, X., Lai, X., Lyu, Y., Zhang, H., Fenghuang, Y., ..., Fang, H. (2021). The changing acceptance of COVID-19 vaccination in different epidemic phases in China: A longitudinal study. Vaccines, 9(3), 191. https://doi.org/10.3390/vaccines9030191

Williams, L., Gallant, A. J., Rasmussen, S., Brown Nicholls, L. A., Cogan, N., Deakin, K., ..., Flowers, P. (2020). Towards intervention development to increase the uptake of COVID-19 vaccination among those at high risk: Outlining evidence-based and theoretically informed future intervention content. British Journal of Health Psychology, 25(4), 1039-1054. https://doi.org/10.1111/bjhp.12468

World Health Organization. (2019). Ten Threats to Global Health in 2019. https://www.who.int/newsroom/spotlight/ten-threats-to-global-health-in-2019. Accessed 9 Apr 2021.

Wong, L. P., Alias, H., Wong, P. F., Lee, H. Y., \& AbuBakar, S. (2020). The use of the health belief model to assess predictors of intent to receive the COVID-19 vaccine and willingness to pay. Human Vaccines \& Immunotherapeutics, 16(9), 2204-2214. https://doi.org/10.1080/21645515.2020.1790279

Wong, M. C., Wong, E. L., Huang, J., Cheung, A. W., Law, K., Chong, M. K., .., Chan, P. K. (2021). Acceptance of the COVID-19 vaccine based on the health belief model: A population-based survey in Hong Kong. Vaccine, 39(7), 1148-1156. https://doi.org/10.1016/j.vaccine.2020.12.083

Zhang, K. C., Fang, Y., Cao, H., Chen, H., Hu, T., Chen, Y., ..., Wang, Z. (2021). Behavioral intention to receive a covid-19 vaccination among chinese factory workers: Cross-sectional online survey. Journal of Medical Internet Research, 23(3), e24673. https://doi.org/10.2196/24673 
Zhu, F. C., Guan, X. H., Li, Y. H., Huang, J. Y., Jiang, T., Hou, L. H., .., Chen, W. (2020). Immunogenicity and safety of a recombinant adenovirus type-5-vectored COVID-19 vaccine in healthy adults aged 18 years or older: A randomised, double-blind, placebo-controlled, phase 2 trial. The Lancet, 396(10249), 479-488. https://doi.org/10.1016/S0140-6736(20)31605-6

\section{Authors and Affiliations}

\section{Tomás Caycho-Rodríguez ${ }^{1}$ D . José M. Tomás ${ }^{2}$. Carlos Carbajal-León ${ }^{1}$. Lindsey W. Vilca ${ }^{3}$. Mario Reyes-Bossio ${ }^{4}$. Claudio Intimayta-Escalante ${ }^{5}$. Andrea Vivanco-Vidal ${ }^{4}$. Daniela Saroli-Araníbar ${ }^{4}$. Renzo Felipe Carranza Esteban ${ }^{6} \cdot$ Michael White $^{7}$}

1 Facultad de Ciencias de la Salud, Universidad Privada del Norte, Av. Alfredo Mendiola 6062, Los Olivos, Lima, Perú

2 Department of Methodology for the Behavioral Sciences, Universidad de Valencia, Valencia, Spain

3 Departamento de Psicología, Universidad Peruana Unión, Lima, Perú

$4 \quad$ Facultad de Psicología, Universidad Peruana de Ciencias Aplicadas, Lima, Perú

5 Sociedad Científica de San Fernando (SCSF), Lima, Perú

6 Universidad San Ignacio de Loyola, Lima, Perú

7 Dirección General de Investigación, Universidad Peruana Unión, Lima, Perú 